\title{
Soft-pair corrections to low-angle Bhabha scattering: YFS Monte Carlo approach
}

\author{
S. Jadach* and M. Skrzypek* \\ Department of Physics and Astronomy, The University of Tennessee, Knoxville, Tennessee 37996-1200 \\ and CERN, Theory Division, CH-1211 Geneva 23, Switzerland \\ B. F. L. Ward \\ Department of Physics and Astronomy, The University of Tennessee, Knoxville, Tennessee 37966-1200 \\ and CERN, Theory Division, CH-1211 Geneva 23, Switzerland \\ and SLAC, Stanford University, Stanford, California 94309
}

(Received 4 October 1996)

\begin{abstract}
We use our recently derived real and virtual infrared functions for soft pairs in QED in the YFS exponentiation framework to develop a Monte Carlo event generator realization of the effects of such pairs in the low-angle Bhabha scattering process at SLC or CERN LEP energies. The respective Monte Carlo realization is incorporated into the event generator BHLUMI 2.30, and explicit Monte Carlo data are presented for the ALEPH LCAL luminometer-type acceptance. Our results confirm our earlier semianalytic estimates of the size of such effects in the SLC or LEP luminosity process and provide an event-by-event view of their manifestations. [S0556-2821(97)03903-9]
\end{abstract}

PACS number(s): 11.15.Bt, 11.15.Tk, 12.15.Ji, 13.10.+q

\section{INTRODUCTION}

Recently, the experimental error on the measurement of the CERN $e^{+} e^{-}$collider LEP or SLAC Linear Collider (SLC) luminosity process has been reduced $[1,2]$ to the regime below $0.1 \%$; significant progress has been made to bring the theoretical error to the same level of precision, where the most recently published theoretical precision is now $0.11 \%$ [3-6]. Accordingly, it is important to remove any significant source of uncertainty on the scale of $0.1 \%$ in either the experimental or theoretical determination of the LEP or SLC luminosity cross section, which is the cross section for low-angle Bhabha scattering. Some of the significant outstanding theoretical contributions to the error on the results in [4-6] are the effects of soft-pair production in the luminosity regime. In this paper, we use our recently derived [7] soft-pair real and virtual infrared functions in the YennieFrautschi-Suura (YFS) [8] exponentiation theoretical framework to achieve a Monte Carlo (MC) realization of these soft-pair effects, on an event-by-event basis. This realization is achieved via the Monte Carlo event generator BHLUMI 2.02 [9] of two of us (S.J. and B.F.L.W.), yielding a new version 2.30, which will be described in detail elsewhere [10].

More specifically, in Table I of $[5,6]$, the contribution of soft pairs to the theoretical uncertainty in the prediction of version 4.03 [6] of BHLUMI is calculated, using leading logarithmic YFS exponentiated methods as published in [11]. From the standpoint of the experimental determination of the possible manifestations of this contribution, it is desirable to have access to the events that contain the soft pairs themselves, in conjunction with the always present multiplephoton radiative effects as well. Only in this way can one be

*Permanent address: Institute of Nuclear Physics, ul. Kawiory 26a, Kraków, Poland, www home page: http://hpjmiady.ifj.edu.pl/ sure of the detector response to such pairs. From the theoretical standpoint, there is the same need to calculate directly, in the realistic detector acceptance, the soft-pair effect in a way that takes into account the interplay of the soft pairs and the attendant multiphoton radiative effects. This will then allow an important crosscheck on the leading logarithmic methods in [11]. In these ways, we are led to develop a Monte Carlo event generator for soft-pair effects in the SLC or LEP luminosity regime.

Our strategy for developing a Monte Carlo realization of the soft-pair effects of interest to us is to follow our Monte Carlo realization of multiple-photon radiation in the same low-angle Bhabha scattering process. Thus, we generate the soft pairs with YFS exponentiation by substituting our softpair virtual and real infrared functions from [7] for the respective YFS photon virtual and real infrared functions in our BHLUMI 2.02 MC event generator [9] (version 4.03 may also be used for this purpose), with a consequent change in the multiple photon phase space to the respective multiple soft-pair phase space; this substitution we do in one branch of the Monte Carlo algorithm while retaining the original multiple-photon YFS exponentiated generation in a parallel branch of the Monte Carlo algorithm. Thus, we retain the interplay between multiphoton and multi-soft-pair radiative effects in low-angle Bhabha scattering and arrive in this way at version 2.30 of BHLUMI. Such a multiple soft-pair radiative $\mathrm{MC}$ is an entirely new theoretical paradigm, affording, as it does, the first ever realistic multiple soft-pair radiative events in which the physical four-vectors of the respective soft pairs are available among the list of final-particle four-vectors; we will illustrate this for the purpose of analyzing the soft-pair contribution to the LEP or SLC luminosity cross section for the ALEPH LCAL [1] detector acceptance.

Our work is organized as follows. In the next section, we describe the basic theoretical framework of our calculations, reviewing where necessary the relevant aspects of the YFS 
theory as it is realized by two of us (S.J. and B.F.L.W.) in [9]. In Sec. III, we present our soft-pair Monte Carlo procedure and thereby arrive at version 2.30 of BHLUMI. In Sec. IV, we present numerical results for the ALEPH luminometer-type acceptances. Section V contains our concluding remarks.

\section{THE MASTER FORMULA}

In this section, we describe the relevant theoretical framework we use to develop our realization of soft-pair effects by Monte Carlo methods. More precisely, the algorithm used in this paper to generate pair emission is based on a certain extension of the YFS scheme, as used in [1,7]. Loosely speaking, we allow the emitted soft real photons of the YFS scheme to be either real photons or virtual photons turning into real pairs. The generic type of the real emission graphs resummed this way is shown in Fig. 1(a). The matching virtual graphs are also taken into account. They are shown generically in Fig. 1(b). In this way we of course do not take into account all of the graphs contributing to the soft limit of pairs production. The graphs of Fig. 1, however, constitute an important class of the corrections: the only graphs both leading-logarithmic (LL) and IR singular. In other words, we do not claim any complete exponentiation of soft pairs, but rather use the YFS approach to sum up the LL and IR dominant graphs. It is not difficult to verify that such an extension of the YFS scheme results simply in replacements of all the $B(\widetilde{B}), S(\widetilde{S})$, and $\beta(\bar{\beta})$ functions by the sums of two components: photonic $(\gamma)$ and fermionic $(f)$ ones. These basic $B_{f}\left(\widetilde{B_{f}}\right)$ and $S_{f}\left(\widetilde{S_{f}}\right)$ functions have been defined and calculated in [7].

It follows that the master formula for the cross section in this work is, in its structure, the same as in [9]:

$$
\begin{aligned}
\sigma= & \sum_{n=0}^{\infty} \sum_{n^{\prime}=0}^{\infty} \frac{1}{n !} \frac{1}{n^{\prime} !} \int \frac{d^{3} q_{2}}{q_{2}^{0}} \frac{d^{3} p_{2}}{p_{2}^{0}} \delta^{(4)}\left(p_{1}+q_{1}-p_{2}-q_{2}-\sum_{i=1}^{n} k_{i}-\sum_{i^{\prime}=1}^{n^{\prime}} k_{i^{\prime}}^{\prime}\right) \exp \left[Y_{\gamma}\left(\Omega_{1}, p_{1}, p_{2}\right)+Y_{\gamma}\left(\Omega_{2}, q_{1}, q_{2}\right)\right. \\
& \left.+Y_{f}\left(p_{1}, p_{2}\right)+Y_{f}\left(q_{1}, q_{2}\right)\right] \int \prod_{i=1}^{n}\left[\frac{d^{3} k_{i}}{k_{i}^{0}} \widetilde{S}_{\gamma}\left(p_{1}, p_{2}, k_{i}\right)\left[1-\Theta\left(\Omega_{1} ; k_{i}\right)\right]+\delta^{(4)}\left(k_{i}-k_{a_{i}}-k_{b_{i}}\right)\right. \\
& \left.\times d^{4} k_{i} \frac{d^{3} k_{a_{i}}}{k_{a_{i}}^{0}} \frac{d^{3} k_{b_{i}}}{k_{b_{i}}^{0}} \widetilde{S}_{f}\left(p_{1}, p_{2}, k_{a_{i}}, k_{b_{i}}\right)\right] \int \prod_{j=1}^{n^{\prime}}\left[\frac{d^{3} k_{j}^{\prime}}{k_{j}^{\prime 0}} \widetilde{S}_{\gamma}\left(q_{1}, q_{2}, k_{j}^{\prime}\right)\left[1-\Theta\left(\Omega_{2} ; k_{j}^{\prime}\right)\right]+\delta^{(4)}\left(k_{j}^{\prime}-k_{a_{j}}^{\prime}-k_{b_{j}}^{\prime}\right)\right. \\
& \left.\times d^{4} k_{j}^{\prime} \frac{d^{3} k_{a_{j}}^{\prime}}{k_{a_{j}}^{\prime 0}} \frac{d^{3} k_{b_{j}}^{\prime}}{k_{b_{j}}^{\prime 0}} \widetilde{S}_{f}\left(q_{1}, q_{2}, k_{a_{i}}^{\prime}, k_{b_{i}}^{\prime}\right)\right]\left[\bar{\beta}_{0_{\gamma}}^{(1)}\left(Q, p_{1}, p_{2}, q_{1}, q_{2}\right)+\sum_{i=1}^{n} \bar{\beta}_{1_{\gamma}}^{(1)}\left(Q, p_{1}, p_{2}, q_{1}, q_{2}, k_{i}\right) / \widetilde{S}_{\gamma}\left(p_{1}, p_{2}, k_{i}\right)\right. \\
& +\sum_{j=1}^{n^{\prime}} \bar{\beta}_{1_{\gamma}^{\prime}}^{(1)}\left(Q, p_{1}, p_{2}, q_{1}, q_{2}, k_{j}^{\prime}\right) / \widetilde{S}_{\gamma}\left(q_{1}, q_{2}, k_{j}^{\prime}\right)+\bar{\beta}_{0_{f}}^{(1)}\left(Q, p_{1}, p_{2}, q_{1}, q_{2}\right) \\
& +\sum_{i=1}^{n} \bar{\beta}_{1_{f}}^{(1)}\left(Q, p_{1}, p_{2}, q_{1}, q_{2}, k_{a_{i}}, k_{b_{i}}\right) / \widetilde{S}_{f}\left(p_{1}, p_{2}, k_{a_{i}}, k_{b_{i}}\right) \\
& \left.+\sum_{j=1}^{n^{\prime}} \bar{\beta}_{1_{f}^{\prime}}^{(1)}\left(Q, p_{1}, p_{2}, q_{1}, q_{2}, k_{a_{j}}^{\prime}, k_{b_{j}}^{\prime}\right) / \widetilde{S}_{f}\left(q_{1}, q_{2}, k_{a_{j}}^{\prime}, k_{b_{j}}^{\prime}\right)\right] \Xi_{\mathrm{MC}}\left(p_{i}, q_{i}, k_{i}, k_{j}^{\prime}\right)
\end{aligned}
$$

where the real soft functions $\widetilde{S}$ are defined by

$$
\begin{gathered}
\widetilde{S}_{\gamma}\left(p_{1}, p_{2}, k\right)=-\frac{\alpha}{4 \pi^{2}}\left(\frac{p_{1}}{k p_{1}}-\frac{p_{2}}{k p_{2}}\right)^{2}, \\
\widetilde{S}_{f}\left(p_{1}, p_{2}, k_{a}, k_{b}\right)=\frac{\alpha^{2}}{4 \pi^{4}}\left(\frac{p_{1}}{p_{1} k}-\frac{p_{2}}{p_{2} k}\right)_{\mu}\left(\frac{p_{1}}{p_{1} k}-\frac{p_{2}}{p_{2} k}\right)_{\nu} \frac{4 k_{a}^{\mu} k_{b}^{\nu}-k^{2} g^{\mu \nu}}{2 k^{4}}, \quad k=k_{a}+k_{b} .
\end{gathered}
$$

The virtual form factor $Y_{f}$ is given by the expression [7] 


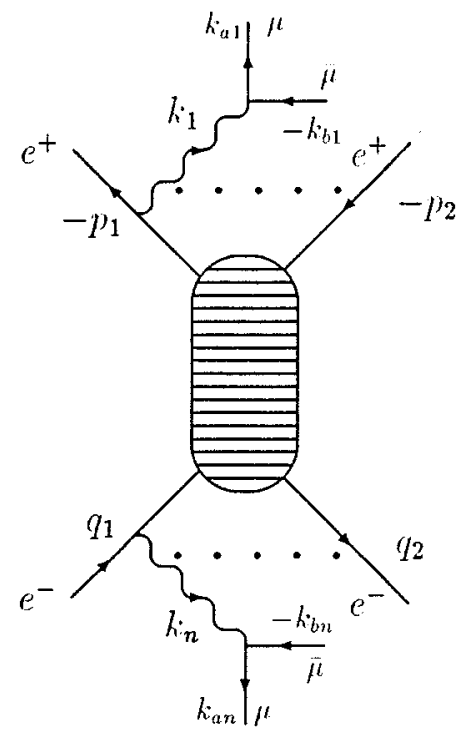

(a)

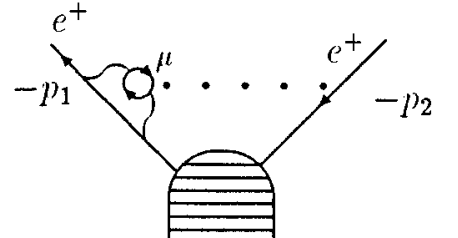

FIG. 1. (a) The class of real emission diagrams resummed in our soft-pair YFS exponentiation calculus. The kinematics is as indicated in the figure; (b) typical virtual corrections included in our soft-pair exponentiation calculus.

$$
\begin{aligned}
& Y_{f}\left(p_{1}, p_{2}\right)=2\left(\frac{\alpha}{\pi}\right)^{2} \operatorname{Re} B_{(\mu)}\left(t_{p}\right)=2\left(\frac{\alpha}{\pi}\right)^{2}\left[-\frac{1}{36} L^{3}+\frac{13}{72} L^{2}+\left(-\frac{1}{36} \pi^{2}-\frac{20}{27}\right) L+C_{B}+\frac{13}{72} \pi^{2}\right], \\
& C_{B}=\left\{\begin{array}{l}
\frac{74}{108}-\frac{2}{6} \frac{\pi^{2}}{6}, \quad \mu=m, \\
-\frac{1}{3} \zeta(3)+\frac{121}{81}-\frac{13}{18} \frac{\pi^{2}}{6}, \quad \mu \gg m,
\end{array}\right. \\
& t_{p}=\left(p_{1}-p_{2}\right)^{2}, \quad L=\ln \frac{\left|t_{p}\right|}{\mu^{2}}, \\
& B_{(\mu)}(s)=e^{2} \int \frac{d^{4} k}{(2 \pi)^{4}} \frac{i P\left(k^{2}\right)}{k^{4}} \frac{1}{2}\left(\frac{2 p_{1}+k}{k^{2}+2 p_{1} k}-\frac{-2 q_{1}+k}{k^{2}-2 q_{1} k}\right)^{2}, \\
& P\left(k^{2}\right)=\frac{\alpha \mu^{2}}{3 \pi}\left[\left(\zeta+\frac{1}{\zeta}-4\right) \frac{1+\zeta}{1-\zeta} \ln \zeta+\frac{5}{3}\left(\zeta+\frac{1}{\zeta}\right)-\frac{22}{3}\right] \\
& s=\left(p_{1}+q_{1}\right)^{2}, \quad \frac{k^{2}}{\mu^{2}}=-\frac{(1-\zeta)^{2}}{\zeta}, \quad k^{2}<0 .
\end{aligned}
$$

From now on $m$ denotes the electron mass, whereas $\mu$ stands for the mass of the additional created fermions. Numerically, only the case $\mu=m$ is important. Let us note that the above-mentioned $Y_{f}$ consist of virtual components only. Because of the finite mass of the pair no additional real IR cutoff is needed and therefore there is no real emission component in $Y_{f}$. Note also that the definition of the $B_{(\mu)}(s)$ function in Eq. (5) is done in the $s$-channel with $p_{1}, q_{1}$ being the incoming $e^{+} e^{-}$fourmomenta. In order to get to Eq. (4) one has to do an analytic continuation to the $t$ channel.

All the photonic functions in Eq. (1) are the same as defined in [9]. For the reader's convenience we collected them also in Appendix A. Finally, the functions $\bar{\beta}_{0,1_{f}}$ corresponding to residual not exponentiated parts of the matrix element are discussed later on and collected in Appendix B.

To conclude the presentation of the master formula we rewrite Eq. (1) in the more compact notation of [9]:

$$
\begin{aligned}
\sigma= & \sum_{n=0}^{\infty} \sum_{n^{\prime}=0}^{\infty} \frac{1}{n !} \frac{1}{n^{\prime} !} \int \frac{d^{3} q_{2}}{q_{2}^{0}} \frac{d^{3} p_{2}}{p_{2}^{0}} \delta^{(4)}\left(p_{1}+q_{1}-p_{2}-q_{2}-\sum_{i=1}^{n} k_{i}-\sum_{i^{\prime}=1}^{n^{\prime}} k_{i^{\prime}}\right) \int \prod_{i=1}^{n}\left(d \omega_{i}^{\gamma}+d \omega_{i}^{f}\right) \\
& \times \int \prod_{i^{\prime}=1}^{n^{\prime}}\left(d \omega_{i^{\prime}}^{\gamma^{\prime}}+d \omega_{i^{\prime}}^{f^{\prime}}\right)\left(\bar{\beta}_{0+1_{\gamma}}+\bar{\beta}_{0+1_{f}}\right) \Xi_{\mathrm{MC}} e^{Y_{\gamma}\left(\Omega_{1}\right)+Y_{\gamma}\left(\Omega_{2}\right)+Y_{f}+Y_{f}^{\prime},}
\end{aligned}
$$


where the radiation distributions $d \omega$ stand for

$$
\begin{aligned}
d \omega_{i}^{\gamma}= & \frac{d^{3} k_{i}}{k_{i}^{0}} \widetilde{S}_{\gamma}\left(p_{1}, p_{2}, k_{i}\right)\left[1-\Theta\left(\Omega_{1} ; k_{i}\right)\right], \\
d \omega_{i}^{f}= & \delta^{(4)}\left(k_{i}-k_{a_{i}}-k_{b_{i}}\right) d^{4} k_{i} \frac{d^{3} k_{a_{i}}}{k_{a_{i}}^{0}} \frac{d^{3} k_{b_{i}}}{k_{b_{i}}^{0}} \\
& \times \widetilde{S}_{f}\left(p_{1}, p_{2}, k_{a_{i}}, k_{b_{i}}\right) .
\end{aligned}
$$

It is worth noting at this point again that, unlike the photonic $d \omega^{\gamma}$, the pair radiation element $d \omega^{f}$ does not have any $\Theta$-type cutoff in the infrared region. For this reason there is no real-pair contribution to the YFS form factor, Eq. (4). This simplification is, as mentioned earlier, possible since pairs have a natural IR cutoff given by their nonzero mass.

\section{THE MONTE CARLO ALGORITHM}

The first thing we do in order to evaluate Eq. (6) into a form suitable for a Monte Carlo algorithm is to replace the true functions $\bar{\beta}_{0+1}+\bar{\beta}_{0+1_{f}}$ with the simple one $b_{0}=\left(2 \alpha^{2} s / t^{2}\right)$. The true $\bar{\beta}_{0+1}$ distribution along with all the other simplifications will be restored by a reweighting procedure in the Monte Carlo algorithm. This simplification allows us to integrate out the $d^{3} k_{a_{i}} d^{3} k_{b_{i}} \delta^{(4)}\left(k_{1}-k_{a_{i}}-k_{b_{i}}\right)$ in Eq. (8), and rewrite it as (cf. [7])

$$
\begin{aligned}
d \omega_{i}^{f}= & -\frac{1}{6 \pi}\left(\frac{\alpha}{\pi}\right)^{2} d^{4} k_{i} \sqrt{1-\frac{4 \mu^{2}}{k_{i}^{2}}} \frac{1}{k_{i}^{2}} \\
& \times\left(1+\frac{2 \mu^{2}}{k_{i}^{2}}\right)\left(\frac{p_{1}}{p_{1} k_{i}}-\frac{p_{2}}{p_{2} k_{i}}\right)^{2} .
\end{aligned}
$$

This form of $d \omega_{i}^{f}$ highly resembles the photonic radiation element $d \omega_{i}^{\gamma}$ of Eqs. (7) and (2). This, in turn, suggests that also for pairs one can use the Sudakov variables, in a manner similar to how they were used for photons in [9]. The main difference is that here, in addition to the $\widetilde{\alpha}_{i}$ and $\widetilde{\beta}_{i}$ variables, we have one more independent variable: $\widetilde{k}_{i}^{2}$, the mass of the pair. In the $p_{1} p_{2}$ rest frame $\left(\mathrm{QRS}_{p}\right.$ denotes this frame) we define

$$
\begin{gathered}
k_{i}^{(0)}=\frac{\sqrt{\left|t_{p}\right|}}{2}\left(\alpha_{i}+\beta_{i}\right), \quad k_{i}^{(3)}=\frac{\sqrt{\left|t_{p}\right|}}{2}\left(-\alpha_{i}+\beta_{i}\right), \\
k_{T}^{2}=\left|t_{p}\right| \widetilde{\alpha}_{i} \widetilde{\beta}_{i}-k_{i}^{2}, \quad k_{i}^{(1)}=k_{T} \cos \phi_{i}, \quad k_{i}^{(2)}=k_{T} \sin \phi_{i}, \\
\widetilde{\alpha}_{i}=\frac{\alpha_{i}}{K_{p}}, \quad \widetilde{\beta}_{i}=\frac{\beta_{i}}{K_{p}}, \quad \widetilde{k_{i}}=\frac{k_{i}}{\sqrt{\left|t_{p}\right| K_{p}}}, \\
K_{p}=\left(1-\sum_{j=1}^{n} \widetilde{\beta}_{j}\right)^{-1} \\
=\frac{p_{1}\left(p_{2}+\sum_{j=1}^{n} k_{j}\right)}{p_{1} p_{2}} .
\end{gathered}
$$

The phase-space limits are given by the following set of inequalities:

$$
0<\widetilde{\alpha}_{i}, \widetilde{\beta}_{i}<1, \quad \widetilde{\alpha}_{i} \widetilde{\beta}_{i} \geqslant \frac{\widetilde{k}_{i}^{2}}{K_{p}} \geqslant \frac{4 \mu^{2}}{\left|t_{p}\right| K_{p}^{2}}, \quad \sum_{i} \widetilde{\beta}_{i}<1, \quad s_{1} \geqslant 0, \quad s_{1}=\left(q_{1}+q_{2}\right)^{2} .
$$

With these variables, the radiation element of Eq. (9) takes the form

$$
d \omega_{i}^{f}=\frac{1}{6 \pi}\left(\frac{\alpha}{\pi}\right)^{2} d{\widetilde{\alpha_{i}}} d \widetilde{\beta}_{i} d \widetilde{k}_{i}^{2} d \phi_{i} \sqrt{1-\frac{4 \mu^{2}}{k_{i}^{2}}}\left(1+\frac{2 \mu^{2}}{k_{i}^{2}}\right) \frac{1}{\widetilde{k}_{i}^{2}} \frac{\widetilde{\alpha}_{i}{\widetilde{\beta_{i}}}_{i}^{2} \widetilde{z}_{i}^{2}}{,}
$$

where $\widetilde{y_{i}}=\widetilde{\beta_{i}}+\left(m^{2} /\left|t_{p}\right|\right) \widetilde{\alpha_{i}}$ and $\widetilde{z_{i}}=\widetilde{\alpha_{i}}+\left(m^{2} /\left|t_{p}\right|\right) \widetilde{\beta}_{i}$. Finally, the master formula (6), rewritten in the Sudakov variables, becomes (see [9] for details of evaluation).

$$
\begin{aligned}
\sigma= & \sum_{n=0}^{\infty} \sum_{n^{\prime}=0}^{\infty} \frac{1}{n !} \frac{1}{n^{\prime} !} \int_{\left|t_{\min }\right|}^{\left|t_{\max }\right|} \frac{d|t|}{s} \int_{0}^{2 \pi} d \phi\left|\frac{t_{p}}{t}\right|\left|\frac{t_{q}}{t}\right| \int \prod_{i=1}^{n}\left(d \omega_{i}^{\gamma}+d \omega_{i}^{f}\right) \\
& \times \int \prod_{i^{\prime}=1}^{n^{\prime}}\left(d \omega_{i^{\prime}}^{\gamma^{\prime}}+d \omega_{i^{\prime}}^{f^{\prime}}\right)\left(\bar{\beta}_{0+1_{\gamma}}+\bar{\beta}_{0+1_{f}}\right) e^{Y_{\gamma}\left(\Omega_{1}\right)+Y_{\gamma}\left(\Omega_{2}\right)+Y_{f}+Y_{f}^{\prime}} .
\end{aligned}
$$

The actual construction of the algorithm is done in the following steps (we will discuss here in detail only the points that are new or different for pairs in comparison to the photonic algorithm of [9]).

(1) The radiation element $d \omega_{i}^{f}$ is simplified to $d \widetilde{\omega}_{i}^{f}$ : 


$$
d \omega_{i}^{f} \rightarrow d \widetilde{\omega}_{i}^{f}=\frac{1}{6 \pi}\left(\frac{\alpha}{\pi}\right)^{2} d \widetilde{\alpha_{i}} d \widetilde{\beta_{i}} d \widetilde{k}_{i}^{2} d \phi_{i} \frac{1}{\widetilde{k_{i}^{2}}} \frac{1}{\widetilde{y_{i}} \widetilde{z_{i}}},
$$

where $\overrightarrow{y_{i}}=\widetilde{\beta_{i}}+\left(\mathrm{m}^{2} / \mathrm{s}\right) \widetilde{\alpha_{i}}$ and $\overrightarrow{z_{i}}=\widetilde{\alpha_{i}}+\left(\mathrm{m}^{2} / \mathrm{s}\right) \widetilde{\beta_{i}}$. Also the phase-space limits are extended to

$$
0<\widetilde{y_{i}}, \widetilde{z_{i}}<1, \quad \widetilde{y_{i}} \widetilde{z_{i}} \geqslant \frac{\widetilde{k_{i}^{2}}}{K_{p \max }}, \quad \widetilde{k_{i}^{2}} \geqslant \frac{4 \mu^{2}}{s}, \quad K_{p, \max }=\frac{s}{\left|t_{\min }\right|} .
$$

The $\left|t_{\min }\right|$ is one of the input parameters of the program. It is discussed at length in [9].

(2) Next, the YFS form factor $Y_{f}+Y_{f}^{\prime}$ is replaced by $2 Y_{f}^{0}$ :

$$
Y_{f}+Y_{f}^{\prime} \rightarrow 2 Y_{f}^{0}=\left(\frac{\alpha}{\pi}\right)^{2} \frac{8}{9} \ln ^{3} \frac{2 \mu \sqrt{\left|t_{p}\right|}}{s} .
$$

(3) Along with similar simplifications for the photonic part of the cross section, as described in [9] (see Appendix A for a brief summary and notation), and the simplification $\bar{\beta}_{0+1_{\gamma}}+\bar{\beta}_{0+1_{f}} \rightarrow b_{0}=\left(2 \alpha^{2} s / t^{2}\right)$ made at the very beginning of the evaluation of Eq. (6), the resulting crude cross section $\sigma_{0}$ becomes remarkably simple:

$$
\sigma_{0}=\sum_{n=0}^{\infty} \sum_{n^{\prime}=0}^{\infty} \frac{1}{n !} \frac{1}{n^{\prime} !} \int_{\left|t_{\min }\right|}^{\left|t_{\max }\right|} \frac{d|t|}{s} b_{0} \int_{0}^{2 \pi} d \phi \int \prod_{i=1}^{n}\left(d \widetilde{\omega}_{i}^{\gamma}+d \widetilde{\omega}_{i}^{f}\right) \int \prod_{i^{\prime}=1}^{n^{\prime}}\left(d \widetilde{\omega}_{i^{\prime}}^{\gamma^{\prime}}+d \widetilde{\omega}_{i^{\prime}}^{f^{\prime}}\right) e^{2 Y_{\Delta}+2 Y_{f}^{0}} .
$$

The above crude cross section is easy to integrate analytically, and yields the result $\sigma_{0}=4 \pi \alpha^{2}\left(1 /\left|t_{\min }\right|-1 /\left|t_{\max }\right|\right)$.

(4) The last step of the algorithm, after all the $p_{1}, p_{2}, q_{1}, q_{2}$, and $k_{i} k_{i}^{\prime},\left(i=1, \ldots, n, i^{\prime}=1, \ldots, n^{\prime}\right)$ momenta are generated, is the generation of the internal degrees of freedom of the pairs, integrated out in Eq. (9). It is done conveniently in the rest frame of each pair $\left(k_{a_{i}} k_{b_{i}}\right)$ with the $z$ axis along the $\left(\mathbf{p}_{2} / p_{2} k-\mathbf{p}_{1} / p_{1} k\right)$ three-vector. The angular distribution there has a form that is easy to generate:

$$
d \sigma_{i} \sim\left[1-\left(1-4 \mu^{2} / k_{a_{i}}^{2}\right) \cos ^{2} \theta_{k_{a_{i}}}\right] d \cos \theta_{k_{a_{i}}} d \phi_{k_{a_{i}}} .
$$

The last issue to be addressed here is the choice of matrix element and the resulting $\bar{\beta}_{0+1_{f}}$ functions. In the present version of the program we use matrix element for one real pair corrections from the graphs shown in Fig. 1(a) only. We neglect the up-down interference, the effect of fermions exchange, the two-photon-emission-type graphs, etc. In other words we keep only those graphs that give both leadinglogarithmic and infrared contributions. As far as the virtual corrections are concerned, only the graphs matching the real ones are included, cf. Fig. 1(b).

The corresponding matrix elements, $\beta_{0_{f}}$ and $\beta_{1_{f}}$, are listed in Appendix B. The more complete matrix element as presented in $[12,13]$ will be implemented elsewhere in our program [14].

\section{RESULTS AND DISCUSSION}

In this section, we illustrate our soft-pair MC with explicit results into the acceptance of LEP/SLC-type luminometers. For definiteness, we consider as an example the ALEPH LCAL acceptance. In all the following we discuss only cor- rections due to the electron pairs, i.e., $\mu=m$ case, and discard all other fermion species.

Specifically, in Figs. 2-4, we show the comparison of our BHLUMI 2.30 MC predictions for the soft-pair effects with the analogous predictions using the LL, semianalytical methods in $[11,15]$. In each figure, we present our MC and LL semianalytical results for the wide $(W)$, narrow $(N)$, and mixed $(M)$ LCAL acceptances $(a, b, c)$ in the standard convention that the angular ranges corresponding to these cuts are $\Theta_{1}$ $<\theta_{e, \bar{e}}<\Theta_{2}$ with $\Theta_{1}=2.7^{\circ}, \Theta_{2}=7.0^{\circ}$ for the wide acceptance; $\Theta_{1}=3.3^{\circ}, \Theta_{2}=6.3^{\circ}$ for the narrow acceptance; for the mixed acceptance it corresponds to one wide and one narrow angular range. The predictions are given as a function of the energy cut $z_{\text {cut }}$ on the variable $z=1-s^{\prime} / s$, which corresponds to the energy fraction of the outgoing clusters into the LCAL acceptances as prescribed in Ref. [1]. For completeness, we always show the results for two different clustering algorithms for the LCAL acceptance, referred to as lcal and $\mathrm{lcal}_{l l}$ in what follows. We will see that our results are relatively insensitive to which version of the LCAL trigger we use. In Fig. 2, we present the results for the pure soft-pair calculations, in which the photonic radiation is turned off, where we show the soft-pair effect as the deviation from unity of the ratio of the cross section with the pairs effect included to that of the respective Born approximation. We see that there is good agreement between the $\mathrm{MC}$ and the $\mathrm{LL}$ semianalytical results for all three acceptances $(W, N, M)$. For definiteness, we note that for the interesting $M$ case, we get for the $z_{\text {cut }}=0.5$ the MC result $-2.7 \times 10^{-4}$ in units of the Born cross section.

In Fig. 3, we continue our LCAL studies with the illustration of the interplay of the soft pairs and the multiple photon radiation by plotting, for all three acceptances, the ratio to the respective Born approximation of the difference between the cross section with both soft-pairs and multiple photon radiation included and the cross section with only 

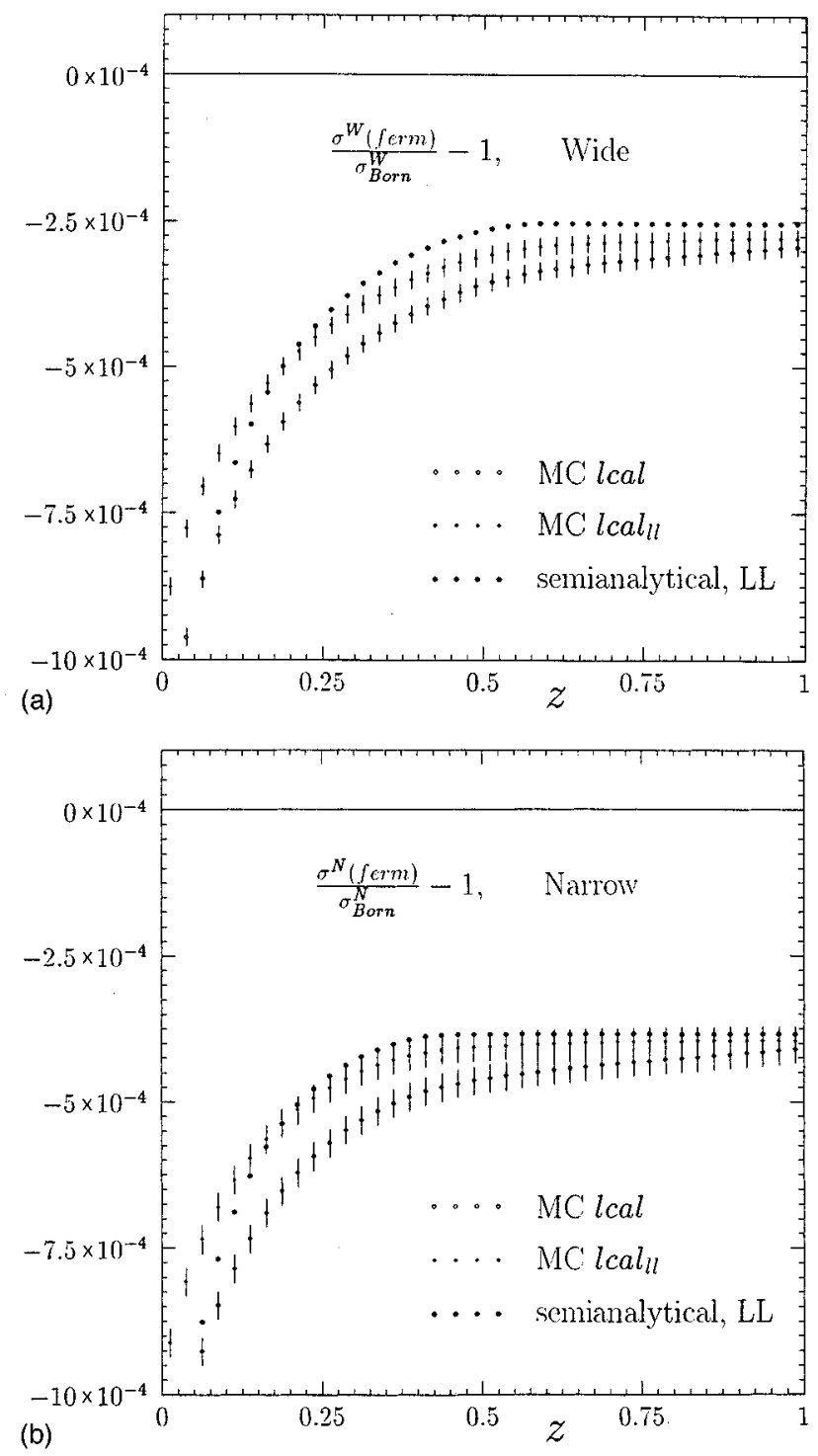

multiple photon radiation included. This we do again as a function of the energy cut $z_{\text {cut }}$. Again, we find agreement with the results in $[11,15]$ and between the LL semianalytical results and the MC. This gives us a good indication of the technical precision of the MC, as we shall discuss presently. Here, we note that in the interesting $M$ case, we get for the $z_{\mathrm{cut}}=0.5$ the $\mathrm{MC}$ result $-1.3 \times 10^{-4}$ in units of the Born cross section.

Turning next to Fig. 4, we look more deeply into the interplay between the soft pairs and multiple photons by plotting the difference from unity of the ratio of the difference between the cross section with both multiple photons and soft pairs included and that with only multiple photons included to the difference between the cross section with only soft pairs included and the respective Born approximation. The deviation of this ratio from unity shows exactly the interplay between soft pairs and multiple photons. Here, the effect is always negative in the MC, i.e., the multiple photon radiation reduces the size of the soft-pairs effects. Intuitively, this is reasonable because, at a minimum, the initial-state radiation reduces the amount of energy available for softpairs creation, so that at least the real soft-pairs effects should be reduced by the multiple photon radiation. Virtual

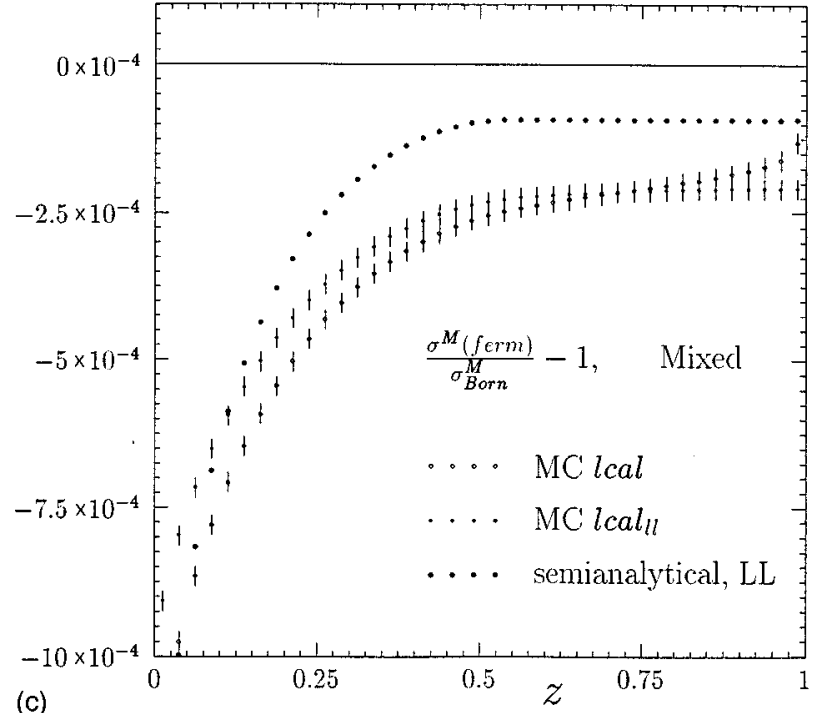

FIG. 2. $\sigma_{f} / \sigma_{B}-1$ for the ALEPH LCAL acceptance, for two different realizations of the clustering algorithm: lcal and $\mathrm{lcal}_{l l}$ (see the text for the details of these acceptances). Here, $\sigma_{f}$ is the respective cross section with soft pairs included as calculated with BHLUMI 2.30 (the open circles and small solid dots for lcal and $\mathrm{lcal}_{l l}$, respectively) and as calculated with our semianalytical LL formulas (the large solid dots) as described in the text. $\sigma_{B}$ is the respective Born cross section. (a) corresponds to the $W$ angular range, (b) corresponds to the $N$ angular range, and (c) corresponds to the $M$ angular range as described in the text. effects are another consideration, however. The LL semianalytical results for the $\mathrm{W}$ and $\mathrm{N}$ cuts are also negative; for the $\mathrm{M}$ cut, there is a region between $z_{\text {cut }}=0.1$ and 0.6 where the multiple-photon effect is positive. Thus, as usual, the naive assessment of real radiation is not sufficient to determine completely the effects of radiative corrections. We note that the LL semianalytical results in Fig. 4 are everywhere within $3 \sigma$ of the $\mathrm{MC}$ results.

This brings us to the discussion of the precision of our results. The conservative estimate we can make is based on a comparison with the LL semianalytical results as we have done, taking the worst case comparison plus an additional $30 \%$ to account for simplifications in matrix element (missing graphs, higher orders, etc.) as an upper limit on the total precision of the MC results. This gives us the basic result $-1.3 \times 10^{-4} \pm 2 \times 10^{-4}$ for the LCAL mixed acceptance at the experimental cut $z_{\text {cut }}=0.5$. We stress that, in addition to the confirmation of the estimates in $[11,15,3]$, our soft-pair MC provides an event-by-event realization of the soft pairs themselves, with the final four-vectors of the pairs available among the list of final-particle four-vectors. Thus, detector simulation of the soft effects may now be effected as necessary. 


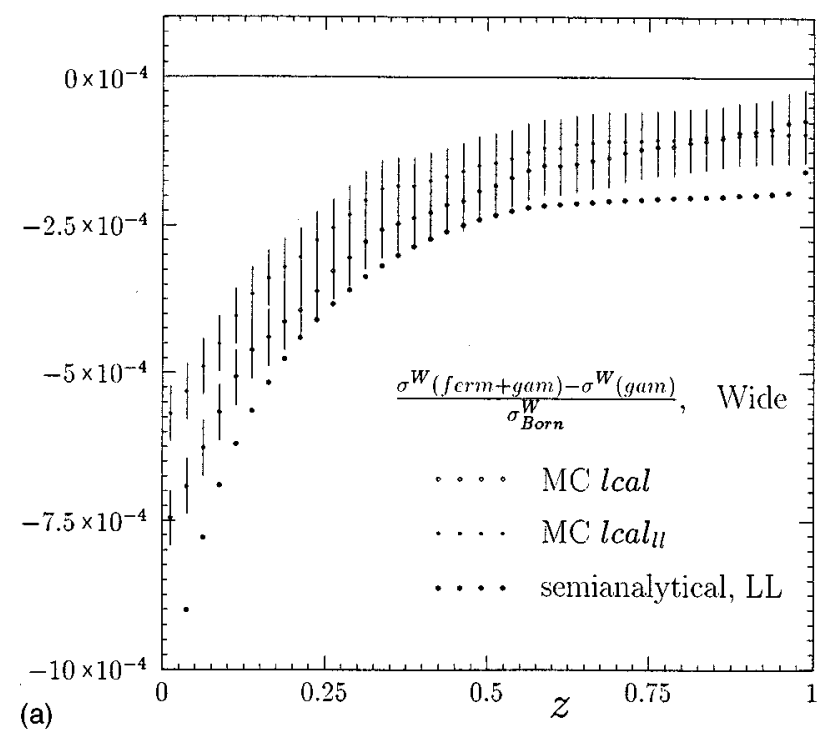

(a)

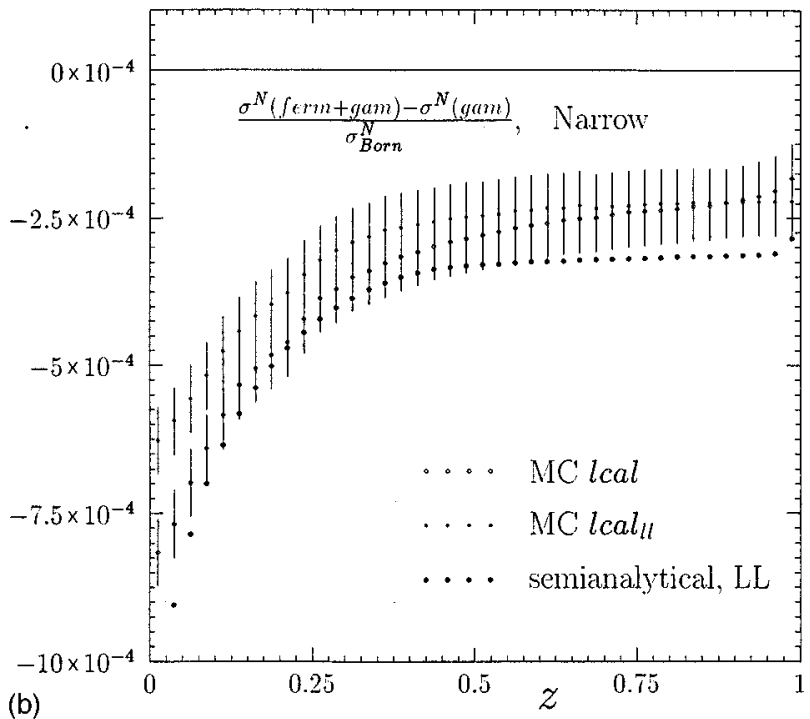

\section{CONCLUSIONS}

In this paper, we have created a new paradigm in theoretical physics: we have created a YFS exponentiated softpairs MC event generator in which the dominant LL and IR singular effects are summed to all orders in $\alpha$. The respective MC event generator is version 2.30 of BHLUMI [9].

We have illustrated our soft-pairs MC realization in the interesting cases of the ALEPH LCAL acceptance. We have computed the soft-pair effects with our MC and compared these $\mathrm{MC}$ results with a LL semianalytical calculation of the type already presented in $[11,15]$. We find good agreement between the two calculations. In addition to corroborating the size of the pairs effects as estimated in [9,6], in determining the precision of BHLUMI 2.02, 4.03, our results illustrate the interplay between multiple photon radiation and soft pairs and provide an event-by-event realization of the softpair effects themselves, in which their four-vectors are available among the list of final-particle four-vectors. Thus, the way is now open to a realistic detector simulation of soft-pair effects in the LEP or SLC luminosity process if it be necessary.

In conclusion, we now have a realistic simulation of the

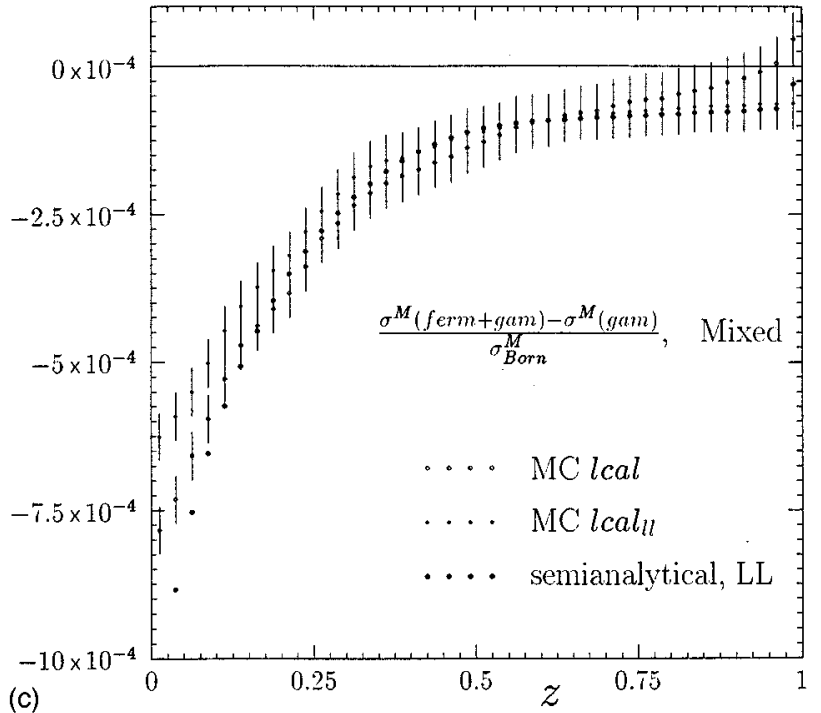

FIG. 3. $\left[\sigma_{f+\gamma}-\sigma_{\gamma}\right] / \sigma_{B}-1$ for the LCAL acceptances described in Fig. 2. Here, $\sigma_{f+\gamma}, \sigma_{\gamma}$ are, respectively, the cross sections with soft pairs and photons and with only multiple photons included and $\sigma_{B}$ is the corresponding Born cross section. As in Fig. 2, the small solid dots (open circles) correspond to calculations done with BHLUMI 2.30 for the lcal $\left(\mathrm{lcal}_{l l}\right)$ versions of the LCAL trigger and the large solid dots correspond to calculations done with our semianalytical LL formulas as described in the text. (a), (b), and (c) correspond to the $W, N$, and $M$ angular ranges as described in the text. soft-pair effects in the LEP or SLC luminosity process and a firm estimate of its total precision. One may now treat soft pairs on an event-by-event basis, in complete analogy with what is done for photons. We find this situation exciting indeed.

\section{ACKNOWLEDGMENTS}

We wish to thank Professor G. Altarelli and Professor G. Veneziano for the kind hospitality and support of the CERN TH Division and wish to thank also the ALEPH Collaboration for its kind hospitality and support. One of us (B.F.L.W.) wishes to thank Professor C. Prescott for the kind hospitality of the SLAC Group A. This work was supported in part by the U.S. Department of Energy Contracts No. DEFG05-91ER40627 and DE-AC03-76ER00515, Polish Government Grants No. EBN 2P30225206 and 2P03B17210.

\section{APPENDIX A}

For the reader's convenience we collect in this appendix the photonic part of the master formula (1) taken from [9]. The YFS form factor is given by 


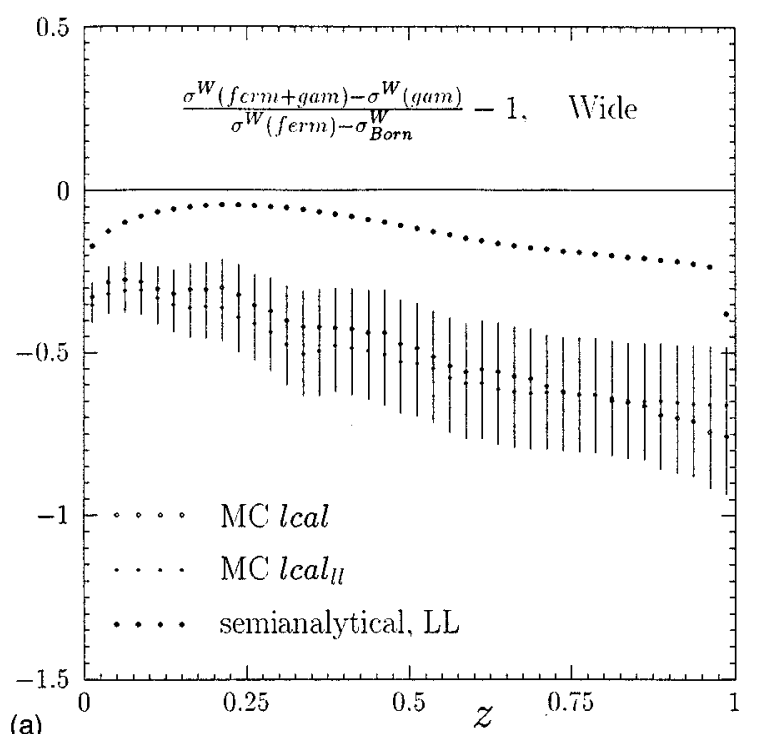

(a)

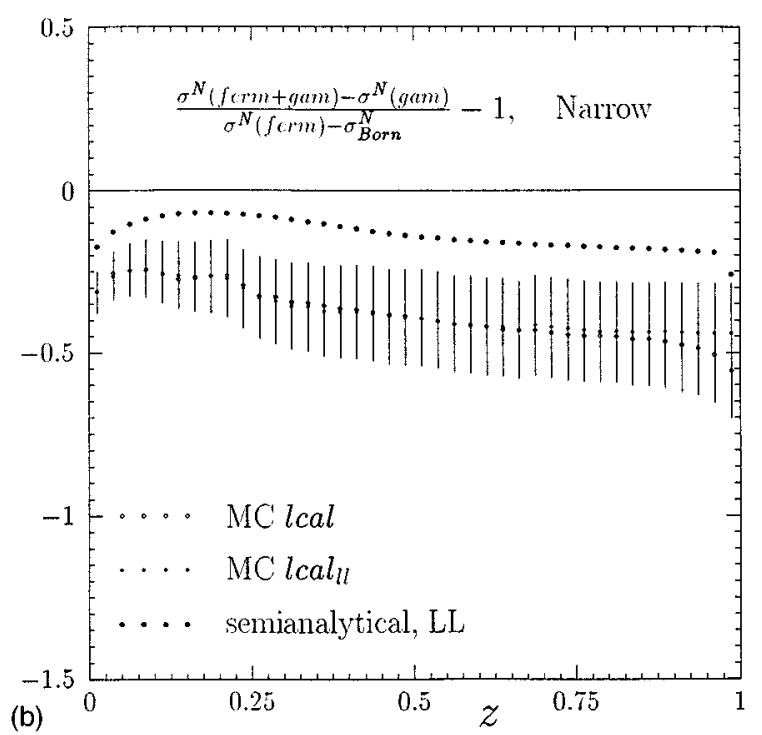

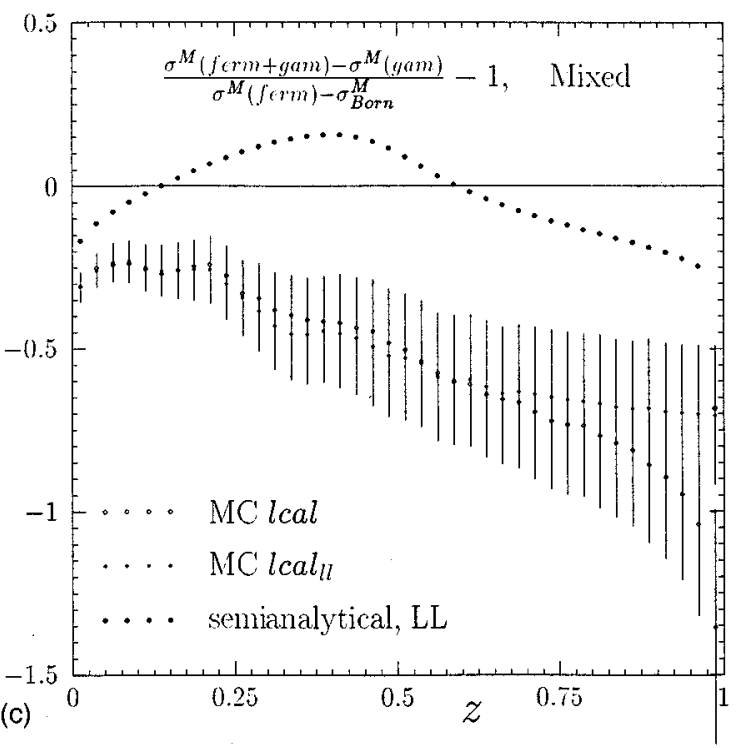

FIG. 4. $\left[\sigma_{f+\gamma}-\sigma_{\gamma}\right] /\left[\sigma_{f}-\sigma_{B}\right]-1$ for the same ALEPH LCAL acceptances as used in Fig. 2. The cross sections $\sigma_{A}, A=f+\gamma, f, \gamma$, and $B$ are as defined in Figs. 2 and 3, and the small solid dots, open circles, and large solid dots have the same correspondence to calculations done with BHLUMI 2.30 for the two LCAL realizations (lcal and $\operatorname{lcal}_{l l}$ ) and with our LL semianalytical formulas as they do in Figs. 2 and 3.

$$
\begin{aligned}
Y_{\gamma}\left(\Omega, p_{1}, p_{2}\right) & =2 \alpha \widetilde{B}\left(\Omega, p_{1}, p_{2}\right)+2 \alpha \operatorname{Re} B\left(p_{1}, p_{2}\right) \\
& =-2 \alpha \frac{1}{8 \pi^{2}} \int \frac{d^{3} k}{k^{0}} \Theta(\Omega ; k)\left(\frac{p_{1}}{k p_{1}}-\frac{p_{2}}{k p_{2}}\right)^{2}+2 \alpha \operatorname{Re} \int \frac{d^{4} k}{k^{2}} \frac{i}{(2 \pi)^{3}}\left(\frac{2 p_{1}-k}{2 k p_{1}-k^{2}}-\frac{2 p_{2}-k}{2 k p_{2}-k^{2}}\right)^{2} .
\end{aligned}
$$

The $\beta_{0,1}$ functions read

$$
\begin{gathered}
\bar{\beta}_{0_{\gamma}}^{(1)}\left(Q, p_{1}, p_{2}, q_{1}, q_{2}\right)=\bar{\beta}_{0_{\gamma}}^{(0)}\left(Q, p_{1}, p_{2}, q_{1}, q_{2}\right)\left(1+2 \delta_{0}+\delta_{\gamma}+\delta_{Z}\right), \\
\delta_{0}=2 \operatorname{Re} F_{1}\left(Q^{2}\right)-2 \alpha \operatorname{Re} B\left(Q^{2}\right)=\frac{1}{2} \beta_{t}, \quad \beta_{t}=2 \frac{\alpha}{\pi}\left(\ln \frac{\left|Q^{2}\right|}{m_{e}^{2}}-1\right), \\
\bar{\beta}_{0_{\gamma}}^{(0)}\left(Q, p_{1}, p_{2}, q_{1}, q_{2}\right)=\frac{2 \alpha_{r}(t)^{2}}{s} \frac{\left(s^{2}+u^{2}+s_{1}^{2}+u_{1}^{2}\right)}{4 t_{p} t_{q}}, \\
\bar{\beta}_{1_{\gamma}}^{(1)}\left(Q, p_{1}, p_{2}, q_{1}, q_{2}, k_{i}\right)=\frac{\alpha_{r}(t)^{2}}{2 s} \frac{\alpha}{4 \pi^{2}} D_{1}^{(1)}\left(Q, p_{1}, p_{2}, q_{1}, q_{2}, k_{i}\right)-\widetilde{S}\left(p_{1}, p_{2}, k_{i}\right) \bar{\beta}_{0_{\gamma}}^{(0)}\left(Q, p_{1}, p_{2}, q_{1}, q_{2}\right), \\
\bar{\beta}_{1_{\gamma}^{\prime}}^{(1)}\left(Q, p_{1}, p_{2}, q_{1}, q_{2}, k_{j}^{\prime}\right)=\frac{\alpha_{r}(t)^{2}}{2 s} \frac{\alpha}{4 \pi^{2}} D_{1^{\prime}}^{(1)}\left(Q, p_{1}, p_{2}, q_{1}, q_{2}, k_{j}^{\prime}\right)-\widetilde{S}\left(q_{1}, q_{2}, k_{j}^{\prime}\right) \bar{\beta}_{0_{\gamma}}^{(0)}\left(Q, p_{1}, p_{2}, q_{1}, q_{2}\right),
\end{gathered}
$$




$$
\begin{gathered}
D_{1}^{(1)}\left(Q, p_{1}, p_{2}, q_{1}, q_{2}, k\right)=\frac{1}{\left(k p_{1}\right)\left(k p_{2}\right)}\left\{\frac{s^{2}+u_{1}^{2}}{\left|t_{q}\right|}\left(1-\frac{2 m_{e}^{2}}{\left|t_{q}\right|} \frac{k p_{1}}{k p_{2}}\right)+\frac{s_{1}^{2}+u^{2}}{\left|t_{q}\right|}\left(1-\frac{2 m_{e}^{2}}{\left|t_{q}\right|} \frac{k p_{2}}{k p_{1}}\right)\right\}, \\
D_{1^{\prime}}^{(1)}\left(Q, p_{1}, p_{2}, q_{1}, q_{2}, k\right)=\frac{1}{\left(k q_{1}\right)\left(k q_{2}\right)}\left\{\frac{s^{2}+u_{1}^{2}}{\left|t_{p}\right|}\left(1-\frac{2 m_{e}^{2}}{\left|t_{p}\right|} \frac{k q_{1}}{k q_{2}}\right)+\frac{s_{1}^{2}+u^{2}}{\left|t_{p}\right|}\left(1-\frac{2 m_{e}^{2}}{\left|t_{p}\right|} \frac{k q_{2}}{k q_{1}}\right)\right\}, \\
t=Q^{2}=\left(p_{2}+\sum_{i=1}^{n} k_{i}-p_{1}\right)^{2}, \quad t_{p}=-2 p_{1} p_{2}, \quad t_{q}=-2 q_{1} q_{2}, \\
s=2 p_{1} q_{1}, \quad s_{1}=2 p_{2} q_{2}, \quad u=-2 p_{1} q_{2}, \quad u_{1}=-2 q_{1} p_{2} .
\end{gathered}
$$

The simplified form of $d \omega_{i}^{\gamma}$ used in actual generation is

$$
d \widetilde{\omega_{i}}=\frac{\alpha}{\pi} \frac{d \widetilde{\alpha_{i}} d \widetilde{\beta_{i}} \theta\left[\max \left(\widetilde{\alpha_{i}}, \widetilde{\beta}_{i}\right)-\Delta\right]}{\left(\widetilde{\alpha_{i}}+\delta_{s} \widetilde{\beta}_{i}\right)\left(\widetilde{\beta}_{i}+\delta_{s} \widetilde{\alpha}_{i}\right)},
$$

with $\Delta$ being the photonic IR cutoff.

Finally, the simplified form factor $Y_{\Delta}$ reads

$$
Y_{\Delta}=2 \frac{\alpha}{\pi} \ln \frac{s}{m^{2}} \ln \Delta
$$

\section{APPENDIX B}

In this appendix we present the one-pair real and virtual matrix elements and corresponding $\beta_{0_{f}}$ and $\beta_{1_{f}}$ functions.

We found the one-pair real cross section resulting from the graphs in Fig. 1(a) (upper line only, single emission) to be

$$
d \sigma_{0 v}^{1 r}=\left(\frac{\alpha}{\pi}\right)^{4} \frac{1}{8 s}\left|M_{0 v}^{1 r}\right|^{2} d \Omega\left(p_{2}, q_{2}, k_{a}, k_{b}\right),
$$

with

$$
\begin{aligned}
&\left|M_{0 v}^{1 r}\right|^{2}= {\left[8 M _ { k } ^ { 2 } \left(2 \mathcal { D } _ { 1 } \mathcal { D } _ { 2 } \left\{2\left(p_{p} k_{m}\right)^{2} t_{q}+8 m_{e}^{2}\left(p_{m} k_{m}\right)^{2}+\left(2 \bar{t}+3 K^{2}\right)\left(t_{q}+4 m_{e}^{2}\right) \bar{t}+2 K^{4} t_{q}-K^{2}\left(12 m_{e}^{2}+8 m_{\mu}^{2}\right) t_{q}\right.\right.\right.} \\
&\left.-2 \bar{t}\left[4 m_{e}^{2} t_{q}-\left(p_{p} q_{p}\right)^{2}-\left(k_{m} q_{p}\right)^{2}+32\left(m_{e}^{2}+m_{\mu}^{2}\right) m_{e}^{2}\right]\right\}-4 \mathcal{D}_{1} \mathcal{D}_{2}\left(\mathcal{D}_{1}+\mathcal{D}_{2}\right)\left[\left(t_{q}+4 m_{e}^{2}\right) \bar{t}+K^{2} t_{q}\right]-K^{2}\left(\mathcal{D}_{1}^{2}+\mathcal{D}_{2}^{2}\right) \\
&\left.\times\left[\left(t_{q}+4 m_{e}^{2}\right) \bar{t}+4 m_{e}^{2} t_{q}\right]-4 K^{2}\left[\mathcal{D}_{1}\left(p_{1} q_{p}\right)+\mathcal{D}_{2}\left(p_{2} q_{p}\right)\right]^{2}+\mathcal{D}_{1} \mathcal{D}_{2}\left(\mathcal{D}_{2}^{2}+\mathcal{D}_{1}^{2}\right)\left(t_{q}+4 m_{e}^{2}\right)+2 \mathcal{D}_{1}^{2} \mathcal{D}_{2}^{2}\left(t_{q}-4 m_{e}^{2}\right)\right) \\
&-32 \bar{t}^{2}\left(t_{q}+4 m_{e}^{2}\right)-128 m_{e}^{2} t_{q} X^{2}+32 m_{\mu}^{2} \mathcal{D}_{1} \mathcal{D}_{2}\left[4\left(k_{p} q_{p}\right)^{2} \bar{t}+\left(\mathcal{D}_{1}-\mathcal{D}_{2}\right)^{2} t_{q}+4 m_{e}^{2}\left(\mathcal{D}_{2}+\mathcal{D}_{1}\right)^{2}\right]-32\left[2\left(p_{1} q_{p}\right) X\right. \\
&\left.\left.-2 \mathcal{D}_{2}\left(k_{p} q_{p}\right)\left(p_{1} k_{m}\right)-\mathcal{D}_{1} \mathcal{D}_{2}\left(k_{m} q_{p}\right)\right]\left[2\left(p_{2} q_{p}\right) X+2 \mathcal{D}_{1}\left(k_{p} q_{p}\right)\left(p_{2} k_{m}\right)-\mathcal{D}_{1} \mathcal{D}_{2}\left(k_{m} q_{p}\right)\right]\right] \frac{1}{\mathcal{D}_{1}^{2} \mathcal{D}_{2}^{2} \overline{t t}_{q} M_{k}^{4}}, \\
& k_{m}=k_{a}-k_{b}, \quad k_{p}=k_{a}+k_{b}, \quad M_{k}^{2}=k_{p}^{2}, \\
& p_{m}=p_{1}-p_{2}, \quad p_{p}=p_{1}+p_{2}, \quad t_{p}=p_{m}^{2}, \\
& q_{m}=q_{1}-q_{2}, \quad q_{p}=q_{1}+q_{2}, \quad t_{q}=q_{m}^{2}, \\
& \mathcal{D}_{1}=-2\left(p_{1} k_{p}\right)+M_{k}^{2}, \quad \mathcal{D}_{2}=2\left(p_{2} k_{p}\right)+M_{k}^{2}, \\
& \bar{t}=t_{p}-M_{k}^{2}+\mathcal{D}_{1}+\mathcal{D}_{2}, \quad K^{2}=M_{k}^{2}+4 m_{e}^{2}+4 m_{\mu}^{2}, \\
& X=\mathcal{D}_{1}\left(p_{2} k_{m}\right)+\mathcal{D}_{2}\left(p_{1} k_{m}\right)
\end{aligned}
$$

and

$$
d \Omega\left(p_{i}, \ldots, k_{j}\right)=\frac{d^{3} p_{i}}{2 p_{i}^{0}} \cdots \frac{d^{3} k_{j}}{2 k_{j}^{0}},
$$




$$
d \sigma_{0 v}^{0 r}=\frac{2 \alpha^{2}}{s t^{2}}\left(s^{2}+u^{2}+s_{1}^{2}+u_{1}^{2}\right) d \Omega\left(p_{2}, q_{2}\right) .
$$

Note that for one pair $\bar{t}=t_{q}=t$, but with some additional emission this is not true any more. From the above cross section we derive the $\beta_{1 f}$ function

$$
\bar{\beta}_{1_{f}}=\frac{d \sigma_{0 v}^{1 r}}{d \Omega\left(p_{2}, q_{2}, k_{a}, k_{b}\right)}-\widetilde{S}_{(\mu)} \frac{d \sigma_{0 v}^{0 r}}{d \Omega\left(p_{2}, q_{2}\right)} .
$$

The virtual corrections of Fig. 1(b) at the one-loop level lead to the formula [16,17]

$$
\begin{gathered}
d \sigma_{(0+1) v}^{0 r}=d \sigma_{0 v}^{0 r}\left[1+4\left(\frac{\alpha}{\pi}\right)^{2} F_{2}^{(\mu)}\right], \\
F_{2}^{(\mu)}=-\frac{1}{36} L^{3}+\frac{19}{72} L^{2}+\left(-\frac{1}{36} \pi^{2}-\frac{265}{216}\right) L+C_{F}+\frac{19}{72} \pi^{2}, \\
L=\ln \frac{-t}{\mu^{2}}, \quad C_{F}=\left\{\begin{array}{l}
\frac{383}{108}-\frac{11}{6} \frac{\pi^{2}}{6}, \quad \mu=m, \\
-\frac{1}{3} \zeta(3)+\frac{3355}{1296}-\frac{19}{18} \frac{\pi^{2}}{6}, \quad \mu \gg m .
\end{array}\right.
\end{gathered}
$$

Finally,

$$
\begin{aligned}
\bar{\beta}_{0_{f}}=\frac{d \sigma_{(0+1) v}^{0 r}}{d \Omega\left(p_{2}, q_{2}\right)}-4\left(\frac{\alpha}{\pi}\right)^{2} \operatorname{Re} B_{(\mu)} \frac{d \sigma_{0 v}^{0 r}}{d \Omega\left(p_{2}, q_{2}\right)}=\frac{d \sigma_{0 v}^{0 r}}{d \Omega\left(p_{2}, q_{2}\right)}\left[1+4\left(\frac{\alpha}{\pi}\right)^{2}\left(\frac{1}{21} L^{2}-\frac{35}{72} L+C_{0}\right)\right], \\
C_{0}= \begin{cases}\frac{103}{36}-\frac{\pi^{2}}{6}, \quad \mu=m, \\
\frac{473}{432}+\frac{1}{6} \frac{\pi^{2}}{6}, \quad \mu \gg m .\end{cases}
\end{aligned}
$$

[1] B. Pietrzyk, in Tennessee International Symposium on Radiative Corrections: Status and Outlook, Gatlinburg, Tennessee, 1994, edited by B. F. L. Ward (World Scientific, Singapore, 1995).

[2] LEP Electroweak Working Group, "A Combination of Preliminary LEP Electroweak Results from the 1995 Summer Conferences, CERN Report No. CERN LEPEWWG/95-02 (unpublished); see also OPAL Report No. ALEPH 95-093 PHYSICS 95-086, DELPH 95-137 PHYS 562, L3 Note 1814, TN312 (unpublished).

[3] S. Jadach, E. Richter-Wa̧s, B. F. L. Ward, and Z. Wąs, Phys. Lett. B 353, 362 (1995); 384, 488 (1996).

[4] Bhaba Working Group, S. Jadach et al. in Physics at LEP2, edited by G. Altarelli, T. Sjöstrand, and F. Zwirner (CERN Report No. 96-01, Geneva 1996), Vol. 2, p. 229.

[5] A. Arbuzov et al., Phys. Lett. B 383, 238 (1996).

[6] S. Jadach et al., "Upgrade of the Monte Carlo program BHLUMI for Bhabha scattering at low angles to version 4.04," CERN Report No. CERN-TH/96-158, UTHEP-96-0601, 1996 (unpublished).
[7] S. Jadach, M. Skrzypek, and B. F. L. Ward, Phys. Rev. D 49, 1178 (1994).

[8] D. R. Yennie, S. Frautschi, and H. Suura, Ann. Phys. (N.Y.) 13, 379 (1961).

[9] S. Jadach, E. Richter-Wa̧s, B. F. L. Ward, and Z. Wạs, Comput. Phys. Commun. 70, 305 (1992).

[10] S. Jadach, M. Skrzypek, and B. F. L. Ward (unpublished).

[11] S. Jadach, M. Skrzypek, and B. F. L. Ward, Phys. Rev. D 47, 3733 (1993).

[12] A. Arbuzov et al., in Tennesee International Symposium on Radiative Corrections: Status and Outlook [1].

[13] A. Arbuzov et al., CERN Report No. CERN-TH/95-313 (unpublished).

[14] S. Jadach et al. (in preparation).

[15] S. Jadach, M. Skrzypek, and M. Martinez, Phys. Lett. B 280, 129 (1992).

[16] G. J. H. Burgers, Phys. Lett. 164B, 167 (1985).

[17] B. A. Kniehl, Phys. Lett. B 237, 127 (1990). 